\title{
P-0625 Association between pancreatic steatosis and diabetic microvascular complications in type 2 diabetes mellitus
}

\author{
H.S. Kwon ${ }^{1}$, S.O. Park ${ }^{2}$, J.S. Jeong ${ }^{1}$, M.K. Kim¹, D.J. Chung ${ }^{3}$, K.H. Baek ${ }^{1}$, K.H. Song \\ ${ }^{1}$ Yeouido St. Mary's Hospital, Internal Medicine, Seoul, Korea. \\ ${ }^{2}$ Gwangmyeong Sungae Hospital, Internal Medicine, Gwangmyeong, Korea. \\ ${ }^{3}$ Yeouido St. Mary's Hospital, Radiology, Seoul, Korea.
}

AIM

Evidence that pancreatic steatosis is related to $\beta$ cell dysfunction and development of type 2 diabetes mellitus is emerging. But, data on the influence of pancreatic steatosis on systemic vascular complication of diabetes mellitus is rare.

In the previous study, we found that pancreatic steatosis was associated with subclinical atherosclerosis in patients with type 2 diabetes mellitus. This finding suggests that pancreatic steatosis might be related to macrovascular complication.

In this study, we hypothesized that pancreatic steatosis could also effect to microvascular complication. So, we investigated whether pancreatic steatosis was associated with diabetic retinopathy (DMR) in patients with type 2 diabetes mellitus.

\section{MATERIALS AND METHODS}

Total one hundred eighty-six consecutive patients with type 2 diabetes mellitus in whom conventional outpatient treatment with either diet or any combination of oral antidiabetic agents had failed were referred to the inpatient diabetes service.

- Obesity was defined as body mass index (BMI) $\geq 25 \mathrm{~kg} / \mathrm{m}^{2}$ according to the Asianspecific BMI cut-off values from the World Health Organization report.

The presence of diabetic retinopathy was assessed by an expert ophthalmologist using dilated fundoscopy.

- Computed tomography (CT) images were acquired using the standard clinical abdominal CT protocol utilizing a multi-slice CT scanner. Pancreatic CT attenuations was measured in three pancreatic regions (head, body and tail) with areas of $1.0 \mathrm{~cm}^{2}$

For assessment of pancreatic steatosis, we used three parameters. First was mean pancreatic CT attenuation (P mean) of three pancreatic regions (head, body and tail). Second was difference between mean pancreatic to splenic attenuation (P-S), Third was ratio of pancreas-to-spleen attenuation $(\mathrm{P} / \mathrm{S})$. All parameters was expressed as Hounsfield unit.

* Kim SY, et al showed that the measurements of P-S $(r=-0.616, \mathrm{P}<0.01)$ and $\mathrm{P} / \mathrm{S}(\mathrm{r}=$ $-0.622, \mathrm{P}<0.01$ ) were correlated to histologic pancreatic fat fraction (Radiology 2014 Apr; 271(1) : 104-12). So we used P-S and P/S for pancreatic steatosis parameters as well as $\mathrm{P}$ mean in this study.

\section{RESULTS}

Table 1. Clinical characteristics in study subjects

\begin{tabular}{|c|c|c|c|c|}
\hline & $\begin{array}{c}\text { Total } \\
(\mathrm{N}=186)\end{array}$ & $\begin{array}{c}\text { Non-obese } \\
(\mathrm{N}=92)\end{array}$ & $\begin{array}{l}\text { Obese } \\
(\mathrm{N}=94)\end{array}$ & $P$ value \\
\hline \multicolumn{5}{|l|}{ Sex } \\
\hline Female, $n(\%)$ & $108(58)$ & $51(55.43)$ & $56(59.57)$ & 0.57 \\
\hline Male, $\mathrm{n}(\%)$ & $78(42)$ & $41(44.57)$ & $38(40.43)$ & 0.57 \\
\hline Age (years)* & $58.29 \pm 14.70$ & $57.9 \pm 14.9$ & $58.6 \pm 14.6$ & 0.76 \\
\hline Smoking, n (yes, \%) & $32(17)$ & $20(21.74)$ & $10(10.64)$ & 0.04 \\
\hline Alcohol, $n$ (heavy dinker, \%) & $53(28)$ & $29(31.52)$ & $22(23.4)$ & 0.21 \\
\hline Hypertension, $\mathrm{n}(\%)$ & $92(49)$ & $33(35.87)$ & $57(60.64)$ & 0.0007 \\
\hline Systolic blood pressure (mmHg)* & $123.47 \pm 14.98$ & $123 \pm 14.6$ & $123.9 \pm 15.4$ & 0.67 \\
\hline Diastolic blood pressure $(\mathrm{mmHg})^{*}$ & $73.89=8.79$ & $73.9 \pm 8.6$ & $73.9 \pm 9.0$ & 0.98 \\
\hline Duration of diabetes (years)* & $14.90 \pm 9.55$ & $15.21 \pm 9.87$ & $14.61 \pm 9.31$ & 0.69 \\
\hline Fasting blood glucose $(\mathrm{mmHg})^{*}$ & $160.50 \pm 52.86$ & $157.7 \pm 54.8$ & $163.3 \pm 50.9$ & 0.47 \\
\hline $\mathrm{HbAlc}(\%)$ & $9.57 \pm 2.09$ & $9.5 \pm 2.3$ & $9.7 \pm 1.9$ & 0.49 \\
\hline Fasting C-peptide (ng/ml)* & $2.93 \pm 2.33$ & $2.43 \pm 1.83$ & $3.35 \pm 2.44$ & 0.004 \\
\hline Stimulated C-peptide (ngml)* & $4.27 \pm 3.04$ & $3.66 \pm 2.54$ & $4.79 \pm 3.01$ & 0.006 \\
\hline Body mass index $\left(\mathrm{kg} / \mathrm{m}^{2}\right)$ & $25.91 \pm 4.12$ & $22.8 \pm 1.7$ & $29.0 \pm 3.4$ & $<.0001$ \\
\hline Waist circumference $(\mathrm{cm})^{*}$ & $92.94 \pm 13.48$ & $83.74 \pm 10.7$ & $100.9=10.1$ & $<.0001$ \\
\hline Total abdominal fat area $\left(\mathrm{cm}^{2}\right)^{*}$ & $347.60 \pm 143.87$ & $259.3 \pm 77.7$ & $436.8 \pm 142.2$ & $<.0001$ \\
\hline Visceral fat area $\left(\mathrm{cm}^{2}\right)^{*}$ & $140.67 \pm 61.48$ & $107.9=46.7$ & $172.8 \pm 58.5$ & $<.0001$ \\
\hline Subcutaneous fat area $\left(\mathrm{cm}^{2}\right)^{*}$ & $186.78 \pm 94.40$ & $141.3 \pm 57.7$ & $235 \pm 100.6$ & $<.0001$ \\
\hline Total cholesterol (mg/dL)* & $175.43 \pm 50.24$ & $175.2 \pm 47.2$ & $175.2 \pm 54.0$ & 0.99 \\
\hline Triglyceride (mg/dl) & $191.29(37-1013)$ & $158.9(37-1013)$ & $223.7(38-941)$ & 0.0009 \\
\hline HDL cholesterol (mg/dL)* & $41.45 \pm 11.98$ & $42.6 \pm 12.2$ & $40.1 \pm 11.8$ & 0.16 \\
\hline LDL cholesterol (mg/dL)* & $94.23 \pm 36.80$ & $95.9 \pm 37.1$ & $93.0 \pm 36.7$ & 0.60 \\
\hline Diabetic retinopathy, n (\%) & $78(42 \%)$ & $38(41.3 \%)$ & $40(42.6 \%)$ & 0.86 \\
\hline
\end{tabular}

The mean duration of diabetes was $14.9 \pm 9.55$ years and mean age was $58.29 \pm$ 14.7 years. The mean body mass index (BMI) was $25.9 \pm 4.1 \mathrm{~kg} / \mathrm{m}^{2}$ and mean $\mathrm{HbA} 1 \mathrm{c}$ was $9.57 \pm 2.09 \%$.

Fasting C-peptide, glucagon stimulated C-peptide, waist circumference, total abdominal fat, visceral fat area, subcutaneous fat area, and triglyceride were more higher in the obese patients than those who were not obese.
Table 2. Characteristics of subjects according to the degree of pancreatic steatosis

\begin{tabular}{lcccccc}
\hline & \multicolumn{7}{c}{ Total } & & \\
\hline & \multicolumn{2}{c}{ P mean } & \multicolumn{2}{c}{ P-S } & \multicolumn{2}{c}{ P/S } \\
\hline & $\mathrm{r}$ & $\mathrm{P}$-value & $\mathrm{r}$ & $\mathrm{P}$-value & $\mathrm{r}$ & P-value \\
\hline \hline Age (years) & -0.293 & $<.0001$ & -0.263 & 0.0002 & -0.269 & 0.0001 \\
Duration of diabetes (years) & -0.233 & 0.0025 & -0.224 & 0.0036 & -0.228 & 0.003 \\
Fasting blood glucose $(\mathrm{mg} / \mathrm{dL})$ & -0.014 & 0.8479 & -0.0334 & 0.6425 & -0.028 & 0.6952 \\
HbA1c $(\%)$ & 0.032 & 0.6584 & 0.0456 & 0.5267 & 0.033 & 0.6437 \\
Fasting C-peptide $(\mathrm{ng} / \mathrm{ml})$ & -0.002 & 0.9765 & 0.006 & 0.9357 & 0.005 & 0.9422 \\
Stimulated C-peptide $(\mathrm{ng} / \mathrm{ml})$ & -0.024 & 0.7404 & -0.017 & 0.8134 & -0.016 & 0.8275 \\
\hline \hline Systolic blood pressure $(\mathrm{mmHg})$ & -0.077 & 0.2865 & -0.053 & 0.4586 & -0.061 & 0.3937 \\
Diastolic blood pressure $(\mathrm{mmmg})$ & -0.025 & 0.7254 & -0.019 & 0.7911 & -0.021 & 0.7726 \\
\hline \hline Body mass index $\left(\mathrm{kg} / \mathrm{m}^{2}\right)$ & -0.011 & 0.8748 & -0.054 & 0.4487 & -0.042 & 0.5624 \\
Waist circumference $\left(\mathrm{cm}^{2}\right)$ & -0.291 & 0.0001 & -0.246 & 0.0014 & -0.260 & 0.0007 \\
Total abdominal fat area $\left(\mathrm{cm}^{2}\right)$ & -0.239 & 0.0007 & -0.200 & 0.005 & -0.217 & 0.0022 \\
Visceral fat area $\left.(\mathrm{cm})^{2}\right)$ & -0.206 & 0.0038 & -0.169 & 0.0176 & -0.175 & 0.0141 \\
Subcutaneous fat area $\left(\mathrm{cm}^{2}\right)$ & -0.078 & 0.2745 & -0.046 & 0.5176 & -0.060 & 0.4023 \\
Log Triglyceride & -0.042 & 0.556 & -0.043 & 0.5518 & -0.038 & 0.5993 \\
HDL cholesterol $(\mathrm{mg} / \mathrm{dL})$ & 0.115 & 0.1074 & 0.082 & 0.2541 & 0.088 & 0.2197 \\
LDL cholesterol $(\mathrm{mg} / \mathrm{dL})$ & 0.011 & 0.8766 & 0.007 & 0.918 & 0.008 & 0.9151 \\
\hline
\end{tabular}

- All pancreatic steatosis parameters were negatively correlated with age $(r=-0.293, P<0.0001)$, duration of diabetes $(r=-0.233, P=0.0025)$, waist circumference $(r=-0.291, P=0.0001)$, total abdominal fat area $(r=-0.239, P=0.0007)$, and visceral fat area $(r=-0.206, P=0.0038)$.

Fig 1. Prevalence of DMR according to the degree of pancreatic steatosis parameters and in total population (left), non-obese and obese subjects (right).
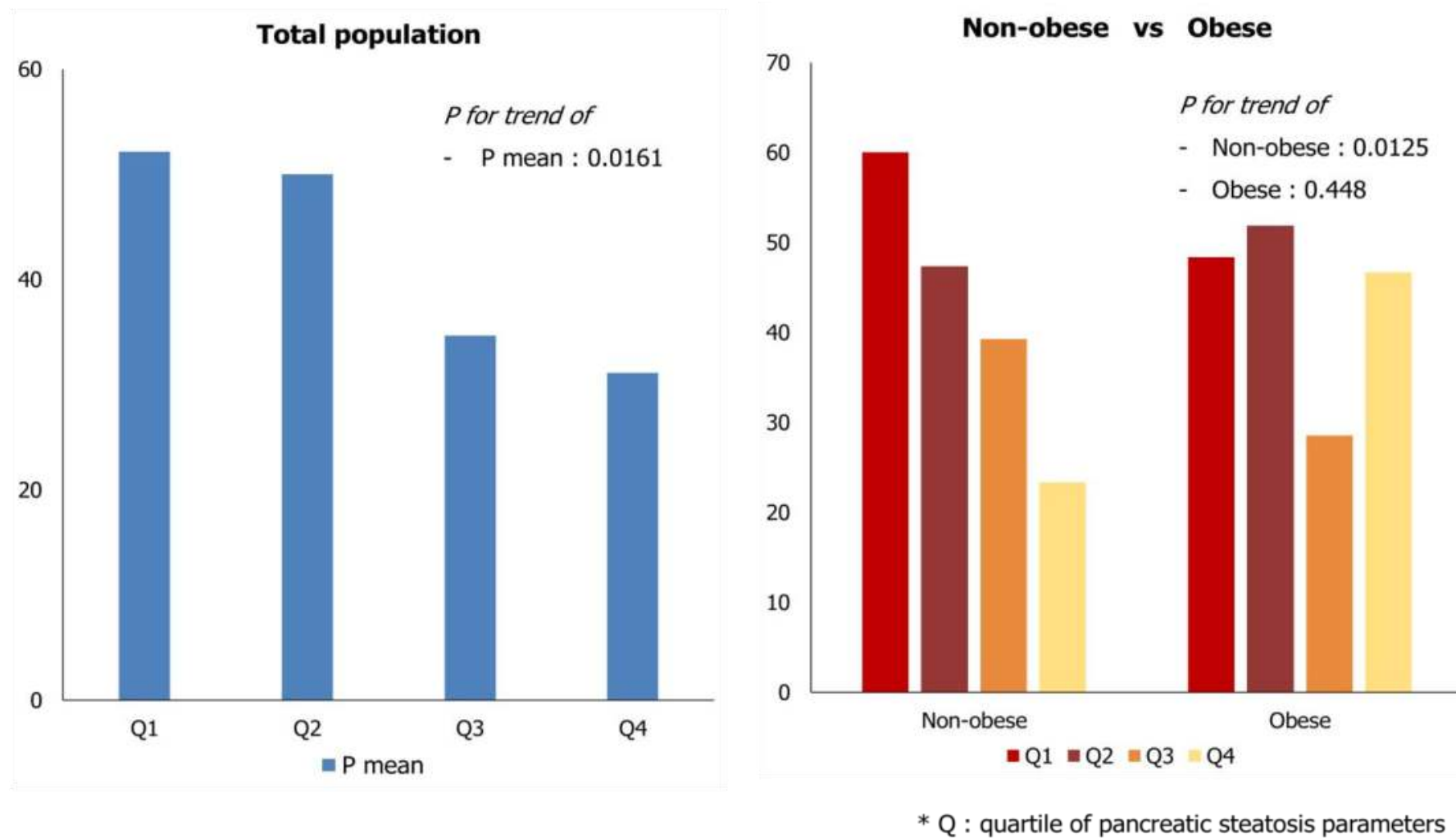

Fig 2. ROC curves of pancreatic steatosis for prevalence of DMR in non-obese patients.
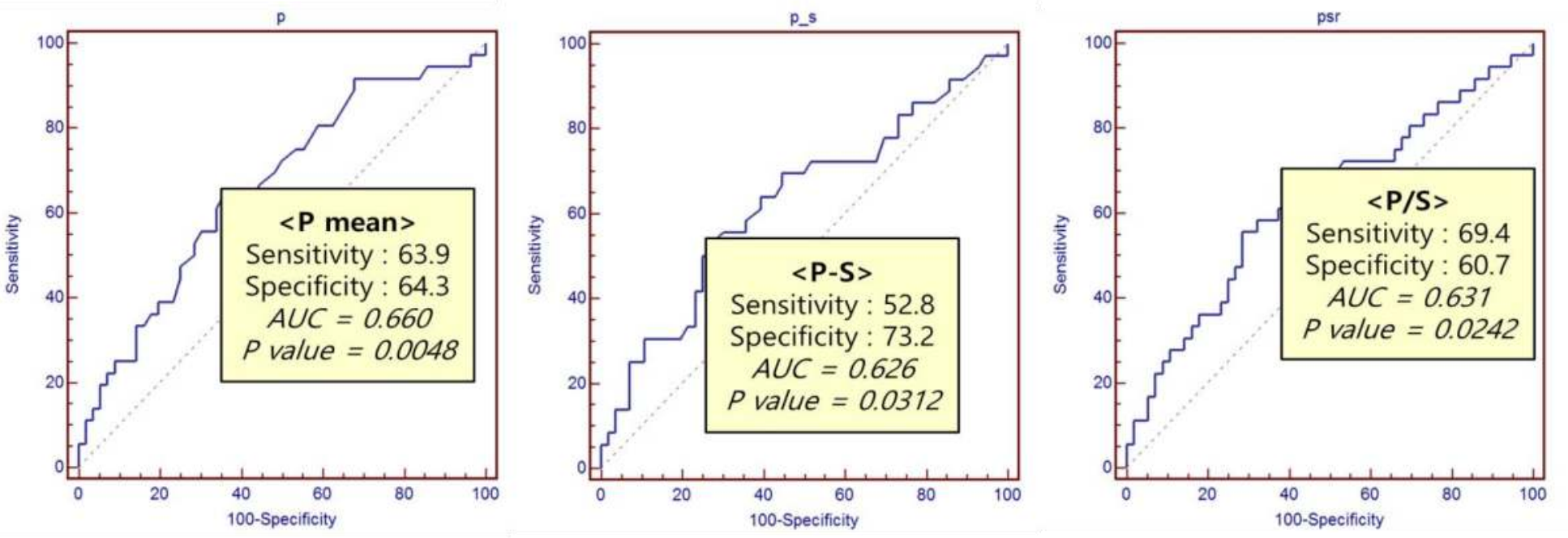

- In the non-obese group, patients with pancreatic steatosis were related to diabetic retinopathy, ( $p$ for trend $=0.0125$ ). But In the obese group, prevalence of diabetic retinopathy was not different according to the degree of pancreatic steatosis. ( $P$ for trend=0.448)

- In the non-obese group, area under the ROC curve (AUC) of P mean for prevalence of DMR was from 0.626 to 0.660 . $P$ value of all parameters was under 0.05

\section{CONCLUSION}

- In conclusion, pancreatic steatosis is significantly associated with DMR in non-obese subjects with type 2 diabetes mellitus.

- $\quad$ Further study will be needed to clarify the mechanism and causal-relationship of pancreatic steatosis in the development of diabetic retinopathy.

\section{ACKNOWLEDGEMENTS}

This research was supported by Basic Science Research Program through the National Research Foundation of Korea (NRF) funded by the Ministry of Education, Science and Technology (NRF-2012R1A1A2007098). 